Cómo citar este artículo: Ortiz-Villota, M. T., Romero-Morales, M. A., \& Meza-Rodríguez, L. D. (2018). La biorremediación con microalgas (Spirulina máxima, Spirulina platensis y Chlorella vulgaris) como alternativa para tratar la eutrofización de la laguna de Ubaque, Colombia. Rev.investig.desarro.innov, 9(1), 163-176. doi: 10.19053/20278306.v9.n1.2018.8153

\title{
La biorremediación con microalgas (Spirulina máxima, Spirulina platensis y Chlorella vulgaris) como alternativa para tratar la eutrofización de la laguna de Ubaque, Colombia
}

\author{
The bioremediation with microalgae (Spirulina maxima, Spirulina platensis y \\ Chlorella vulgaris) as an alternative to treat the eutrophization of the Ubaque \\ lagoon, Colombia
}

\author{
María Teresita Ortiz-Villota' \\ María Angélica Romero-Morales ${ }^{2}$ \\ Laura Daniela Meza-Rodríguez ${ }^{3}$
}

Recibido: mayo 15 de 2018

Aceptado: junio 28 de 2018

\section{Resumen}

La investigación consiste en el tratamiento biológico exsitu de la laguna de Ubaque (Cundinamarca-Colombia) mediante la aplicación del método de biorremediación con las cepas de microalgas: Spirulina máxima, Spirulina platensis y Chlorella vulgaris, con el objetivo de disminuir los niveles de nitratos, nitritos y fosfatos ( $\mathrm{NO}_{3}-, \mathrm{NO}_{2}-, \mathrm{PO} 4-3$ ) de esta fuente hídrica, y así poder comprobar la capacidad depuradora de las microalgas. Para evaluar los efectos de la biorremediación, se utilizó un tipo de diseño experimental "Compuesto Central Factorial 22" realizado con el programa Design-Expert; las cepas fueron adaptadas a las condiciones de la laguna y posteriormente cultivadas de acuerdo a las especificaciones del diseño. Se determinó que la biorremediación con microalgas en la laguna de Ubaque es una alternativa viable para disminuir el nivel de eutrofización; sin embargo, no todas las cepas presentaron resultados significativos.

Palabras clave: biorremediación, eutrofización, laguna, nutrientes, microalgas.

\begin{abstract}
The research consists in the ex-situ biological treatment of the lagoon of Ubaque, Cundinamarca-Colombia, by means of the application of the bioremediation method with the strains of microalgae: Spirulina maxima, Spirulina platensis and Chlorella vulgaris, with the objective of reducing the levels of nitrates, nitrites and phosphates (NO3 -, NO2 -, $\mathrm{PO} 4-3$ ) in this water source, and then, determine the purification capacity of the microalgae. To evaluate the effects of bioremediation, an experimental design type (Central Composite Factorial 22 design) was used with the Design-Expert program; the strains were adapted to the conditions of the lagoon and subsequently cultivated according to the design specifications. It was determined that bioremediation with microalgae in the lagoon of Ubaque is a viable alternative to reduce the level of eutrophication; however, not all strains showed significant results.
\end{abstract}

Keywords: bioremediation, eutrophication, lagoon, nutrients, micoalgae.

1 Licenciada en Biología y Química, Doctora en Biología, Universidad Libre, Bogotá, Colombia. E-mail: mariat.ortizv@unilibre.edu.co

2 Estudiante de Ingeniería Ambiental, Universidad Libre, Bogotá, Colombia. E-mail: mariaa.romerom@unilibrebog.edu.co

3 Estudiante de Ingeniería Ambiental, Universidad Libre, Bogotá, Colombia. E-mail: laurad.mezar@unilibrebog.edu.co Rev.investig.desarro.innov, 9(1), julio-diciembre de 2018, XX-XX. ISSN: 2027-8306 


\section{Introducción}

En la actualidad, los cuerpos de agua superficiales, en su gran mayoría, presentan una alteración en su calidad a causa de los vertimientos domésticos e industriales, que influyen en la salud humana y en los aspectos socioeconómicos de las poblaciones aledañas (Rodríguez, Serna \& Sánchez, 2016). Se estima que anualmente en Colombia se vierten 117.000 toneladas de nitrógeno total y 29.400 toneladas de fósforo a los sistemas hídricos, situación que afecta negativamente la riqueza hídrica del país (MADS, 2016). Surge así la necesidad de idear diferentes estrategias para abordar los desafíos de la calidad del agua en las distintas regiones. En este sentido, la biorremediación es una estrategia eficaz para tratar los altos niveles de nutrientes de las aguas contaminadas, la cual es de un bajo costo con respecto a otras tecnologías que se utilizan para tratar aguas residuales (Rawat, Ranjith, Mutanda \& Bux, 2011).

La biorremediación se fundamenta en la capacidad natural que poseen determinados microorganismos para incorporar contaminantes en sus procesos metabólicos y usarlos como fuente de energía o carbono (Dzionek, Wojcieszyńska \& Guzik, 2016; Mosa, Saadoun, Kumar, Helmy \& Dhankher, 2016). Dentro de los microorganismos utilizados en los tratamientos de biorremediación se encuentran las microalgas, las cuales han sido universalmente conocidas como una alternativa eficaz de tratamiento para la remoción de nutrientes como el nitrógeno y fósforo (Bermeo, 2011).

Las microalgas también se emplean para la eliminación de sustancias tóxicas, como: pesticidas, herbicidas y metales pesados, entre otros; esto gracias a su capacidad de acumular importantes concentraciones de compuestos tóxicos, sin afectar su actividad biológica. Otro beneficio de las microalgas es su tendencia de liberar $\mathrm{O} 2$ por medio de la fotosíntesis, degradando los compuestos orgánicos y generando una acumulación de biomasa, la cual puede ser reutilizada en sistemas de producción de biocombustibles y/o en procesos de avicultura, acuicultura o agricultura (Mehrabadi, Craggs \& Farid, 2015).
La laguna de Ubaque es un cuerpo de agua natural ubicado en el municipio de Ubaque, Cundinamarca, localizada a $4^{\circ} 29^{\prime} 59.25^{\prime \prime} \mathrm{N}$ y $73^{\circ} 56^{\prime} 89^{\prime \prime} \mathrm{W}$ y 2017 m.s.n.m. Según la clasificación Caldas-Lang, esta laguna es una zona fría-húmeda y presenta una humedad relativa del $80 \%$, representando uno de los principales cuerpos de agua del municipio de Ubaque, por su importancia ecológica, cultural y económica (Carmona \& Cruz, 2014). De acuerdo con la investigación de Castellanos y Charry (2016), la laguna presenta diversas problemáticas ambientales, como: eutrofización, la colmatación por sedimentos, la presencia de olores fétidos, la pérdida de espejo de agua y el cambio de color, debido a la proliferación de macrófitas como la Elodea (Elodea canadensis) y la Flor de Loto (Nelumbo lutea) a causa de las cantidades altas de nutrientes como fosfatos y nitratos provenientes de vertimientos de aguas residuales de los predios aledaños y el inadecuado manejo de los residuos por parte de los turistas.

A pesar de la situación identificada en la laguna, actualmente no se encuentran estudios que proporcionen alternativas de solución a la contaminación ni para la preservación de dicho ecosistema. En este sentido, la presente investigación tiene como objetivo disminuir los niveles de contaminación por $\mathrm{NO} 3$ -, NO2 - y PO4-3 en el agua de la laguna de Ubaque, mediante el proceso de biorremediación con cepas de microalgas Spirulina máxima (Sm), Spirulina platensis $(S p)$ y Chlorella vulgaris (Cv), a nivel de laboratorio.

\section{Materiales y métodos}

La figura 1 muestra la relación entre las etapas llevadas a cabo para el desarrollo de la investigación. La primera etapa consistió en la caracterización del agua con el fin de obtener el nivel de nutrientes, posteriormente se llevó a cabo el cultivo y adaptación de las microalgas a las condiciones de la laguna para finalmente realizar el montaje del diseño experimental con las cepas adaptadas; así, se pudo evaluar la eficacia de las tres cepas de microalgas como biorremediadoras en la laguna de Ubaque. 


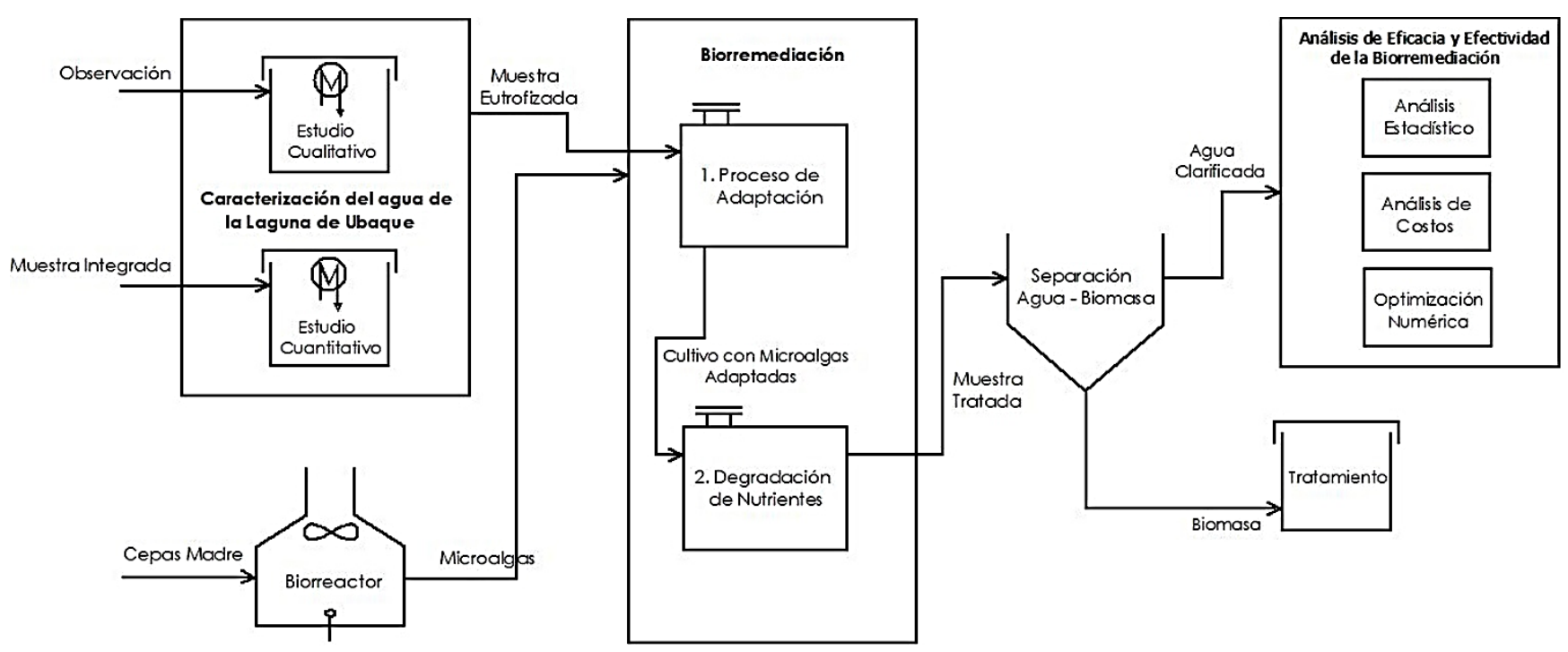

Figura 1. Etapas desarrolladas para la aplicación del proyecto de investigación.

El diseño experimental utilizado en la investigación fue de tipo factorial. Estos diseños son los más eficientes para estudiar el efecto producido por dos o más factores sobre una función respuesta (Ilzarbe, Tanco, Viles \& Álvarez, 2007), pues no solo permiten conocer el efecto individual de cada factor sino también el efecto conjunto de los mismos (Ávila, 2006). Por su potencia y sencillez, el diseño factorial posee un campo de aplicación muy amplio (Medina \& López, 2011), como consecuencia, varios estudios similares al presente como los de Álvarez (2014) y Bolaños y Martínez (2016), lo han preferido para el desarrollo de sus investigaciones.

\subsection{Caracterización del agua}

Con el fin de cuantificar los nutrientes de la laguna, se tomó una muestra integrada, la cual se obtiene a partir de la mezcla de muestras puntuales tomadas de diferentes puntos simultáneamente (IDEAM, 2017). Los puntos de muestreo se visualizan en la figura 2. El agua captada se mantuvo a $4^{\circ} \mathrm{C}$, en frasco de vidrio ámbar, de acuerdo a lo establecido en la guía PT0093 del IDEAM (2018), donde se establecen los parámetros para la toma y preservación de la muestra. 


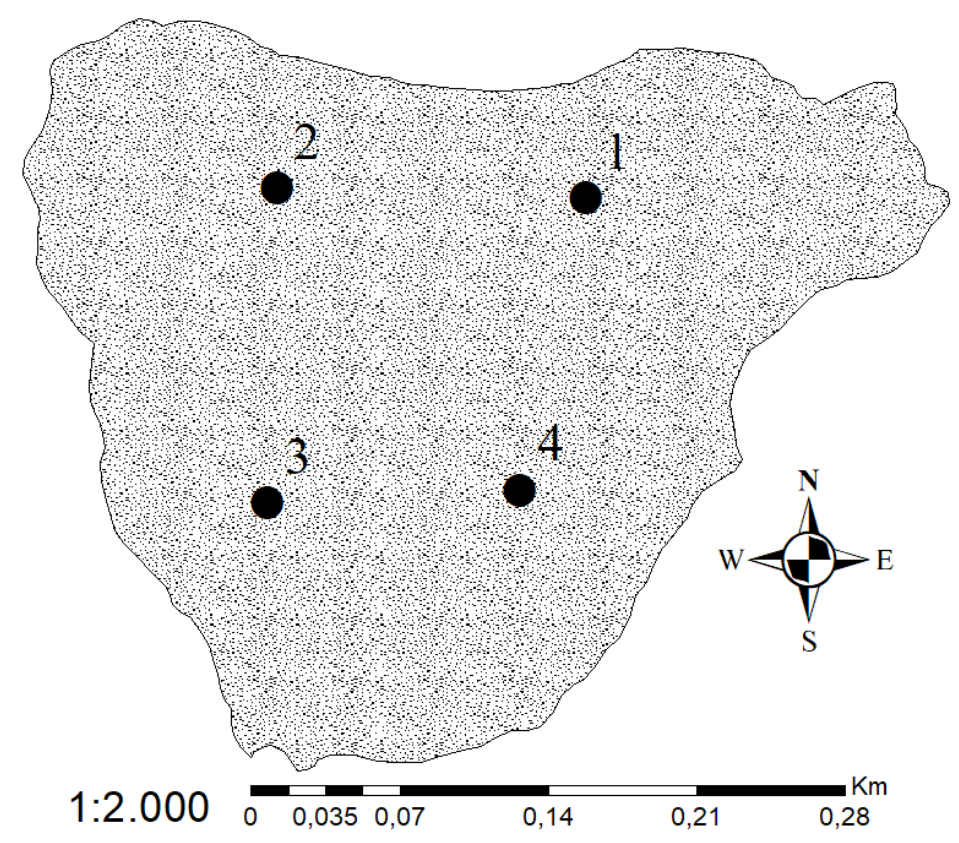

Figura 2. Puntos de muestreo - Laguna de Ubaque.

Las muestras fueron analizadas en el Laboratorio de la Universidad Libre, Bogotá D.C.; se les determinaron los parámetros $\mathrm{NO} 3$-, $\mathrm{NO} 2$ - y $\mathrm{PO} 4-3$ de acuerdo a los métodos estándar establecidos por el IDEAM (2018), indicados en la tabla 1.

\begin{tabular}{lll} 
Nutriente & Método & Equipo utilizado \\
\hline NO3 - & 14TP0092-Nitrato en Agua por Espectrofotometría Ultravioleta & $\begin{array}{l}\text { Espectrofotómetro UV-VIS, marca } \\
\text { MERCK Pharo 300 Spectroquant. }\end{array}$ \\
NO2 - & PT0093-Nitrito en Aguas por Método Colorimétrico & \\
PO4-3 & $\begin{array}{l}\text { TP0094-Fósforo reactivo disuelto en agua por el método del } \\
\text { ácido ascórbico }\end{array}$ & $\begin{array}{l}\text { Laboratorio de calibración: Global } \\
\text { Scientific S.A.S }\end{array}$ \\
\hline
\end{tabular}

Tabla 1. Métodos para la determinación de nutrientes.

\subsection{Cultivo y adaptación de las microalgas}

La siguiente etapa del proceso abarca el cultivo y mantenimiento de las microalgas. Los géneros fueron seleccionados por su capacidad de adaptación, generación de biomasa aprovechable y resistencia (Rodríguez et al., 2017; Weizhi, Yating,
Yizhan \& Haixia, 2017; Forero, Montenegro, Pinilla \& Melgarejo, 2015; Cartagena \& Malo, 2017). Las cepas fueron cultivadas a nivel de laboratorio, en tres fotobiorreactores abiertos de 10 litros. La tabla 2 muestra las condiciones de cultivo, establecidas con base en las recomendaciones de proyecto Malgas (2013). 


\begin{tabular}{ll}
\hline Condiciones & Materiales y Especificaciones \\
\hline Esterilización & Ultrasonido Jeken PS-80 \\
& Frecuencia Ultrasonido: $40 \mathrm{~Hz}$ \\
& Tiempo de esterilización: $30 \mathrm{~min}$ \\
& Potencia de Ultrasonido: $80 \mathrm{~W}$ \\
& Temperatura: $20^{\circ} \mathrm{C}$ \\
& Fotoperiodos $12 \mathrm{~h}$ luz / $12 \mathrm{~h}$ oscuridad \\
Luz Natural & Calefactor Samurai Cerámico eco \\
Temperatura & Graduación: $20 \pm 0.2^{\circ} \mathrm{C}$ \\
& Motor de aire Resun AC-9908 \\
Aireación & Aireación permanente por burbujeo \\
& Flujo de aire: $10 \mathrm{l} /$ min \\
& Voltaje: $100 \mathrm{~V}$ \\
& Chlorella vulgaris: medio Bristol. \\
Medios de cultivo & Spirulina: medio Zarrouk. \\
\hline
\end{tabular}

Tabla 2. Condiciones de cultivo.

Las cepas cultivadas fueron sometidas a un proceso de adaptación durante una semana. Los parámetros controlados se indican en la tabla 3 , los cuales fueron obtenidos de la caracterización de la laguna realizada por Castellanos y Charry (2016).
Durante todo el proceso se realizó el seguimiento a las concentraciones celulares, determinadas mediante el conteo con la cámara Sedgewick Rafter para la cepa Spirulina y la cámara de Neubauer para la Chlorella vulgaris.

\begin{tabular}{ll}
\hline Parámetro & Valor \\
\hline Temperatura media del agua & $20.5^{\circ} \mathrm{C}$ \\
$\mathrm{pH}$ & 7.3 \\
Condiciones Climatológicas & \\
Temperatura media & $17^{\circ} \mathrm{C}$ \\
Humedad Relativa & $80 \%$ \\
\hline
\end{tabular}

Tabla 3. Características Climatológicas y Fisicoquímicas de la Laguna.

\subsection{Diseño experimental}

El diseño experimental, tabla 4, fue realizado con el programa Design-Expert, versión 6.0 .8 portable. Se utilizó un diseño experimental Compuesto Central Factorial 22 con 5 repeticiones del punto central y 4 puntos axiales; los puntos centrales se replican para proporcionar una excelente capacidad de predicción cerca del centro del espacio factorial (Desing Expert, 2018); los factores que se manejaron fueron tiempo de retención (días) y concentración de la cepa (V/V). 


\begin{tabular}{lll}
\hline Prueba & Tiempo (días) & Concentración Cepa (V/V) \\
\hline 1 & 1.00 & 5.00 \\
2 & 3.00 & 23.11 \\
3 & 5.00 & 5.00 \\
4 & 3.00 & 12.50 \\
5 & 3.00 & 12.50 \\
6 & 3.00 & 12.50 \\
7 & 3.00 & 12.50 \\
8 & 1.00 & 20.00 \\
9 & 3.00 & 12.50 \\
10 & 0.17 & 12.50 \\
11 & 5.00 & 20.00 \\
12 & 3.00 & 1.89 \\
13 & 5.83 & 12.50 \\
\hline
\end{tabular}

Tabla 4. Diseño Experimental.

Transcurrido el tiempo de adaptación, cada cepa se cultivó de acuerdo a las especificaciones del diseño experimental; tanto para la fase de adaptación como para el montaje de las pruebas se realizaron tres réplicas. La concentración del inóculo inicial de cada especie fue de $0.5 \times 106$ células / $\mathrm{mL}$, determinada por conteo celular.

\section{Resultados y discusión}

\subsection{Concentración de nutrientes}

En la tabla 5 se presentan las concentraciones de nutrientes encontrados en el agua de la laguna de Ubaque en ppm (mg nutriente /litro de agua).

\begin{tabular}{lllll}
\hline \multicolumn{1}{c}{ Nutriente } & & \multicolumn{3}{c}{ Concentración (mg/l) } \\
& Punto 1 & Punto 2 & Punto 3 & Punto 4 \\
& & & & \\
\hline Nitratos (NO3-) & 0.6 & 0.6 & 0.5 & 0.7 \\
Nitritos (NO2-) & 0.6 & 0.7 & 0.6 & 0.8 \\
Fosfatos (PO4-3) & 0.07 & 0.08 & 0.07 & 0.08 \\
\hline
\end{tabular}

Tabla 5. Concentración de nutrientes.

De acuerdo con la Universidad Tecnológica de Panamá (2006) en aguas superficiales bien oxigenadas, el nivel de nitrito no suele superar $0.1 \mathrm{mg} / \mathrm{l}$, valores entre 0.1 a 0.9 pueden presentar problemas de toxicidad; asimismo, los valores por encima de $1.0 \mathrm{mg} / \mathrm{l}$ son totalmente tóxicos y representan un impedimento en el desarrollo de la vida piscícola y el establecimiento de un ecosistema fluvial en 
buenas condiciones. La concentración de Nitritos obtenida en este caso fue de $0.8 \mathrm{mg} / \mathrm{l}$, el cual está en el rango de toxicidad para ecosistemas acuáticos.

Así mismo, en los trabajos de Moreta (2008) y Pozo (2011), se concluye que nitrógeno y fósforo son en conjunto las causas principales de eutrofización. Las concentraciones elevadas de nitrógeno pueden promover el desarrollo, mantenimiento y proliferación de los productores primarios (fitoplancton, algas bentónicas, macrófitos), contribuyendo al muy extendido fenómeno de la eutrofización cultural de los ecosistemas acuáticos. En la tabla 5 se evidencia que los nutrientes se encuentran en valores considerablemente altos para un cuerpo de agua lentico, como lo es la laguna bajo estudio. Esto ha generado el crecimiento de macrófitas, como la Elodea (Elodea canadensis) y Flor de Loto (Nelumbo nucifera), plantas indicadoras de eutrofización (Zehnsdorf, Hussner, Eismann, Ronicke \& Melzer, 2015; Carbiener, Trémolieres, Mercier \& Ortscheit, 1990).

\subsection{Adaptación de las microalgas}

La figura 3 muestra los resultados del proceso de adaptación para las tres cepas. El conteo celular diario permitió obtener la gráfica de la cinética de crecimiento.

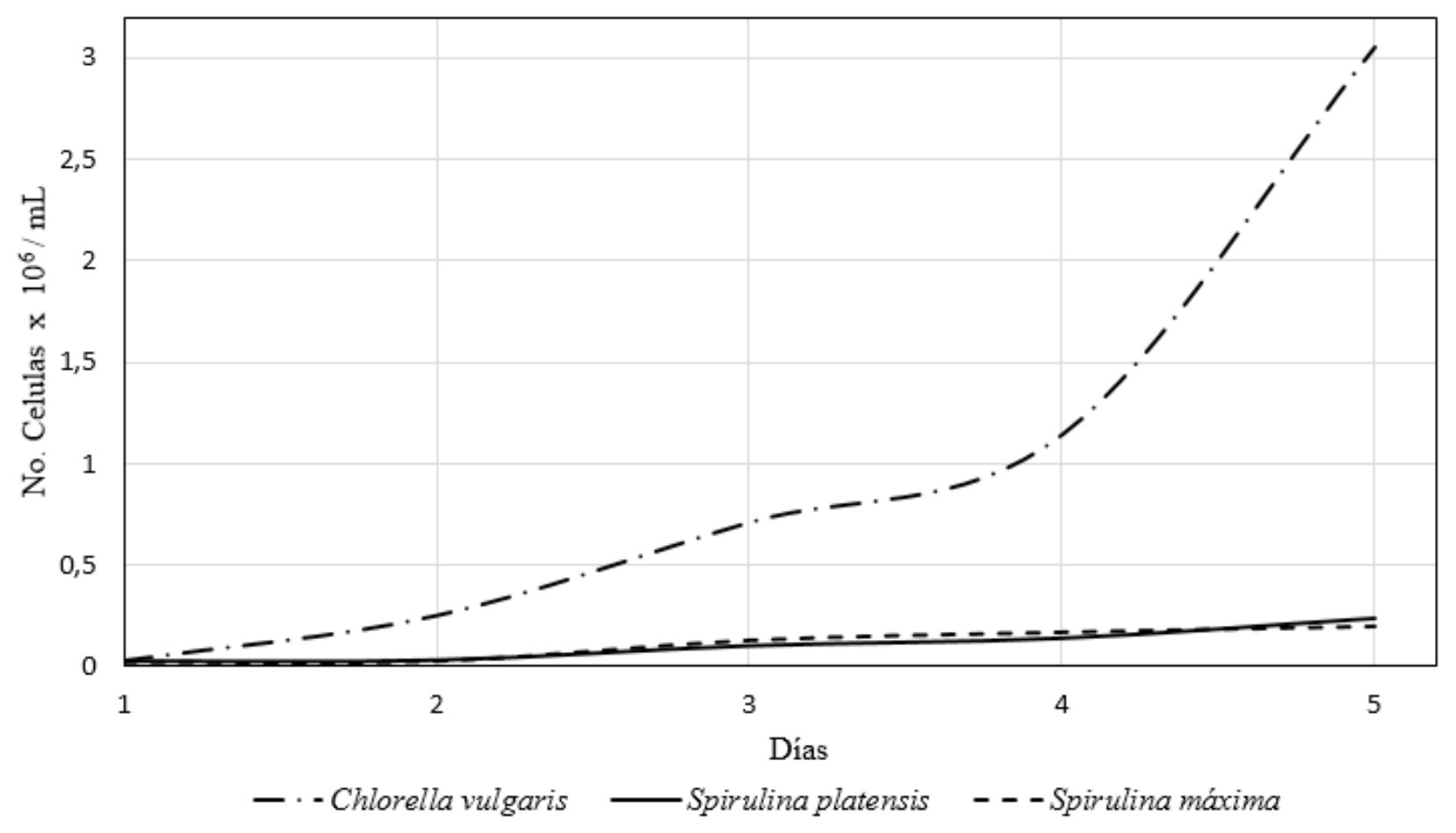

Figura 3. Crecimiento celular.

Los resultados demuestran que la cepa Chlorella vulgaris presenta una cinética de crecimiento mayor a la registrada por la Spirulina; este comportamiento puede deberse a la gran adaptabilidad que tiene la $C v$ a las condiciones de la laguna por ser un alga nativa de Colombia. Lo anterior se diferencia del género Spirulina, cuyo crecimiento presentó limitaciones en la fase de adaptación; esto se atribuye a las características de su lugar de origen, el Lago Chad, África, donde la temperatura 
promedio es de $37^{\circ} \mathrm{C}$, la cual es superior a los 20,5 ${ }^{\circ} \mathrm{C}$ presentes en la laguna de Ubaque.

En cuanto a la Chlorella vulgaris, los resultados se asemejan a los de Bolaños y Martínez (2016), en los cuales se obtuvo un rápido crecimiento exponencial de la cepa $C v$ hacia los primeros 5 días del experimento, en presencia de un medio enriquecido con nitrógeno.

En las tres réplicas llevadas a cabo del periodo de adaptación, el comportamiento del crecimiento de las tres cepas no varió de manera significativa, manteniendo siempre la misma tendencia presentada en la figura 3.

\subsection{Remoción de nutrientes}

\section{Remoción de nitratos}

La figura 4 muestra el comportamiento de las tres cepas al remover Nitratos; como se observa, la CV presenta una mayor afinidad con este nutriente.
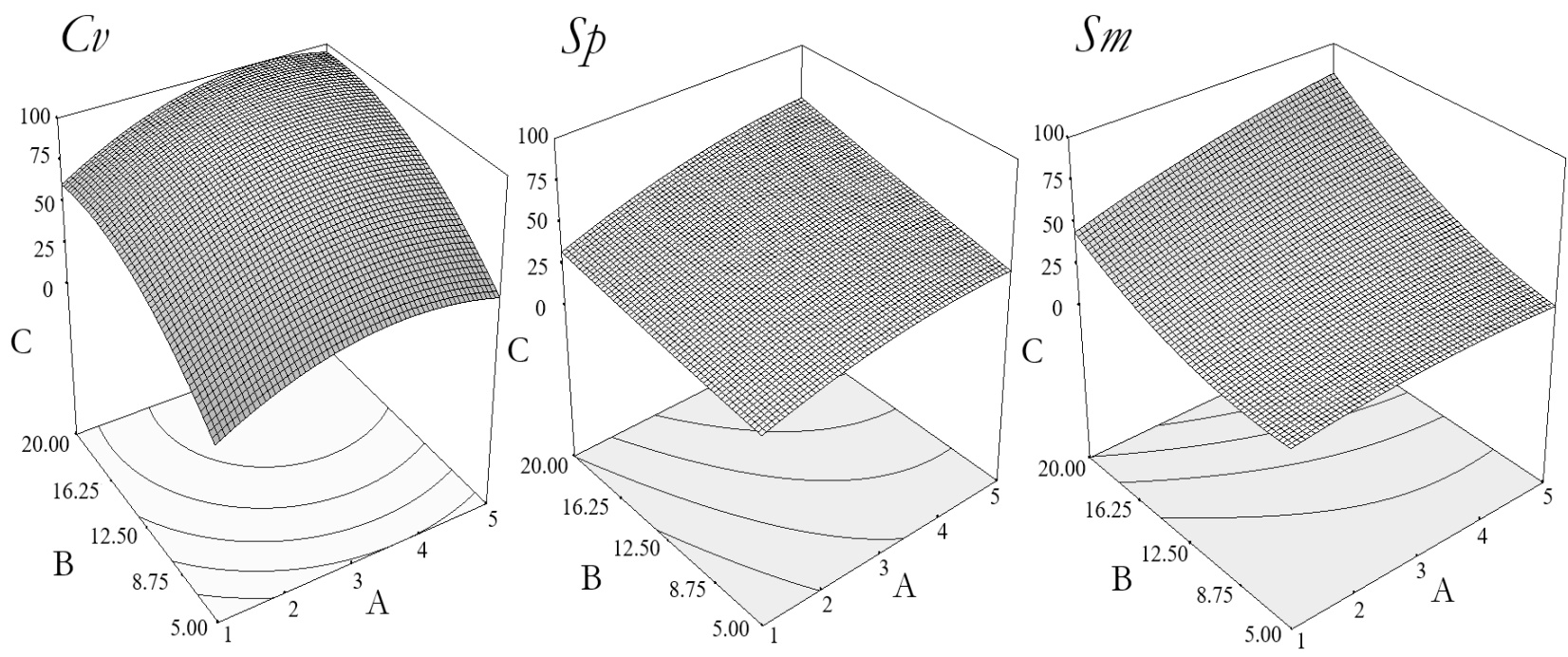

Figura 4. Remoción de Nitratos. A: tiempo de retención (días), B: concentración (V/V) de microalga, C: \% de remoción. Cv: ChloreIla vulgaris, Sp: Spirulina platensis, Sm: Spirulina máxima.

Con la cepa Cv se obtuvo un porcentaje de remoción máximo del $88.24 \%$, alcanzado en el cuarto día del tratamiento; a partir de este momento los valores de remoción no aumentaron significativamente, lo que permite inferir que la biorremediación en la laguna con la cepa $C V$ no requiere un tiempo de retención mayor a cuatro días. En el caso de las Spirulina se reportaron valores máximos de remoción de $85.9 \%$ para la cepa $S m$, y $64 \%$ para la cepa $S p$, porcentajes que fueron alcanzados a un tiempo de cinco días. Las diferencias en los tiempos de retención entre las tres cepas permiten deducir que la $C v$ no solo es la más eficaz para remover nitratos, sino que también es la más eficiente por remover a tiempos de retención menores.

\section{Remoción de nitritos}

En la figura 5 se observan las superficies de respuesta de remoción de nitritos para cada cepa; al igual que con los nitratos, la Cv mostró el mejor comportamiento como biorremediadora. 

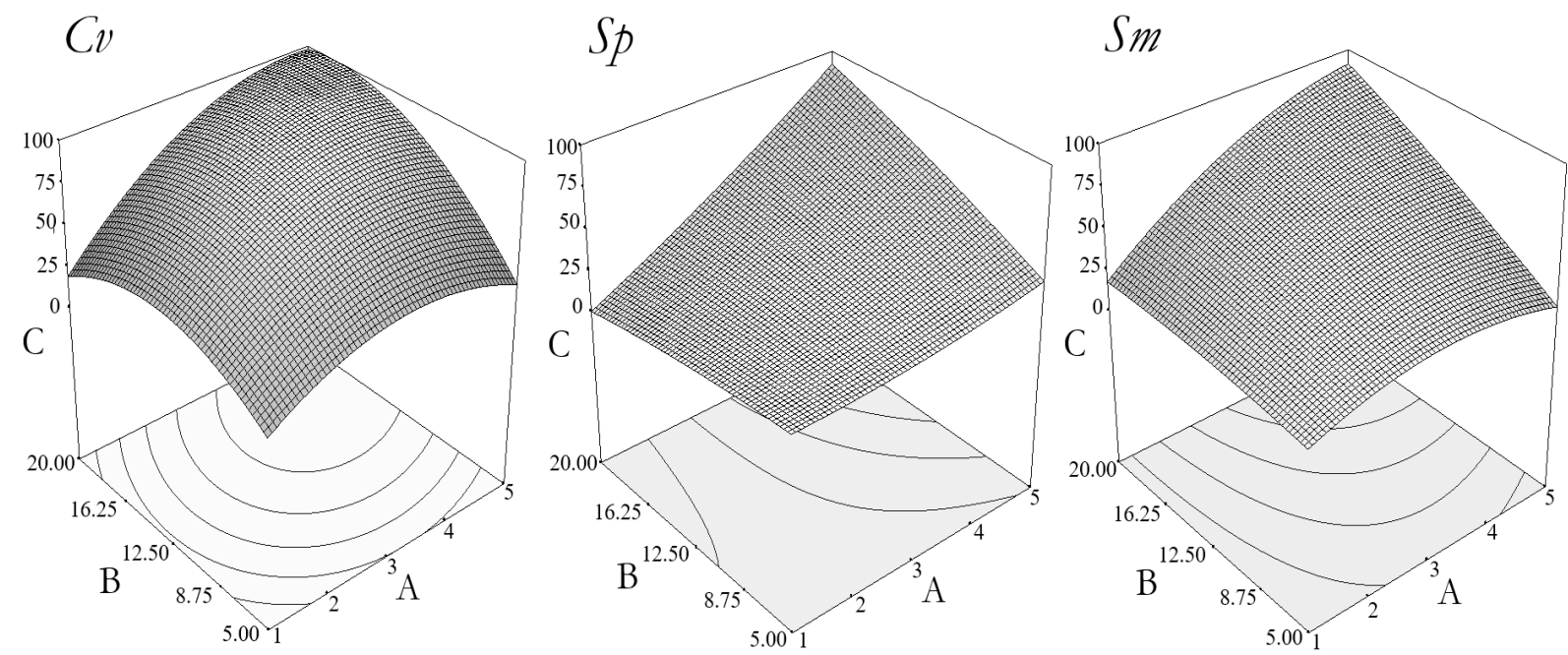

Figura 5. Remoción de Nitritos. A: tiempo de retención (días), B: concentración (V/V) de microalga, C: \% de remoción. Cv: Chlorella vulgaris, Sp: Spirulina platensis, Sm: Spirulina máxima.

El mejor porcentaje de remoción de nitritos se obtuvo con la $C V$, con la cual se alcanzó una remoción de $87.2 \%$, a diferencia de las cepas Spirulina las cuales no lograron superar el $82 \%$; en las tres cepas la máxima remoción se alcanzó al final del experimento. Como se observa en las tres curvas de la figura 5, la remoción no varía de manera significativa en el tiempo cuando se aplica una baja concentración de cepa; esto demuestra que gráfi- camente el factor concentración es más influyente en la remoción de nitritos que el factor tiempo.

\section{Remoción de fosfatos}

El comportamiento de las cepas para la remoción de fosfatos se evidencia en la figura 6; para este nutriente, con las cepas Cv y Sm se obtuvieron porcentajes óptimos de remoción.
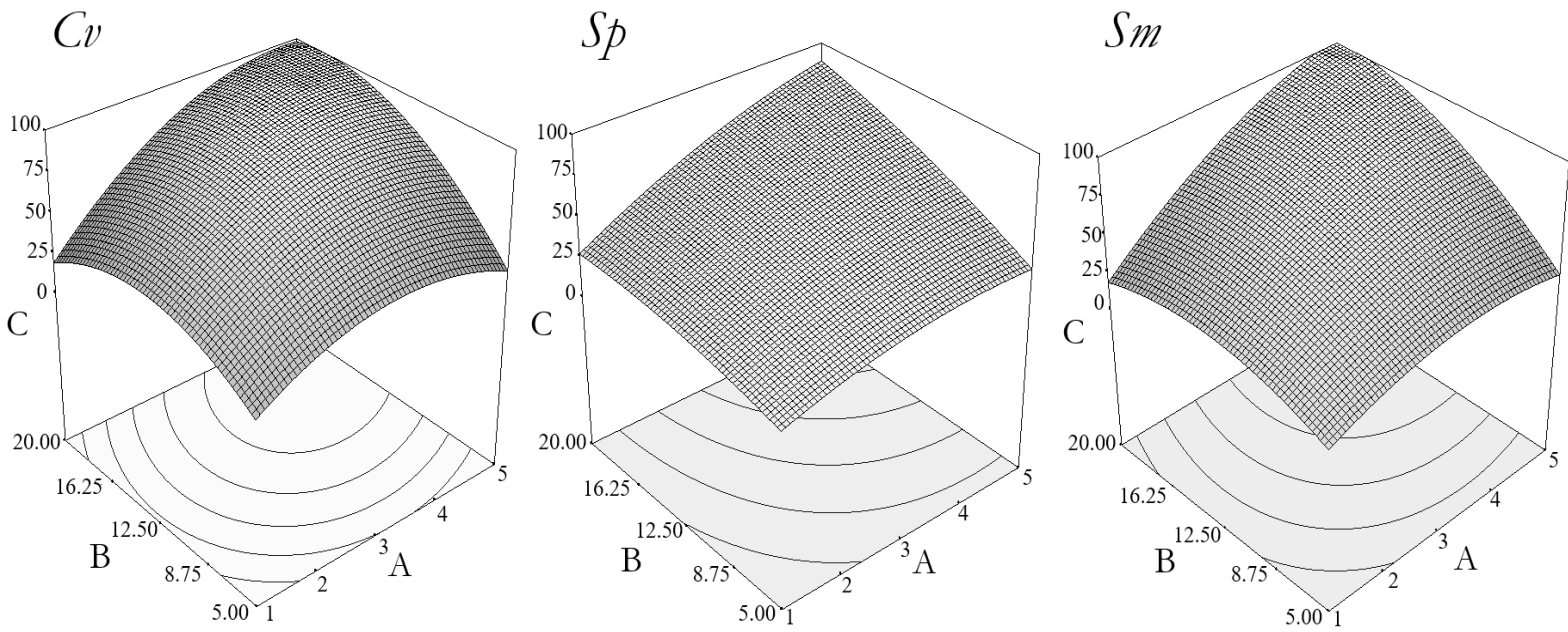

Figura 6. Remoción de Fosfatos. A: tiempo de retención (días), B: concentración (V/V) de microalga, C: \% de remoción. Cv: ChloreIla vulgaris, Sp: Spirulina platensis, Sm: Spirulina máxima. 
Los valores de remoción alcanzados por las cepas Cv y Sm superan el $90 \%$, demostrando ser eficaces biorremediadoras de fosfatos. Por otro lado, la cepa $S p$ logró una remoción de $84 \%$, a pesar de ser un porcentaje alto y significativo no es la mejor opción para remover fosfatos en la laguna.

En las tres curvas de remoción de fosfatos de la figura 6, así como en las superficies de respuesta de nitratos y nitritos, se observa una marcada relación entre los dos factores evaluados, obteniéndose las condiciones más óptimas a tiempos de retención mayores y concentraciones altas.

El comportamiento de la $C v$ frente a cada uno de los nutrientes se asemeja al reportado por Ramos y Pizarro (2018). Los resultados pueden deberse a la capacidad que posee esta microalga para soportar altas concentraciones de nutrientes contenidos en aguas eutrofizadas; además, su metabolismo es muy activo y resistente a cambios ambientales (Blanch, Cárdenas, Durnin, Herrera \& Ortiz, 2017).

En cuanto a las cepas del género Spirulina, aun cuando presentaron inhibición del crecimiento, produjeron cambios adaptativos suficientes para remover los nutrientes en el agua. Sin embargo, los porcentajes de remoción fueron inferiores en comparación con la cepa $C v$, lo cual puede deberse a diversos factores, como el nivel de estrés que tuvieron que soportar frente a la concentración alta de nutrientes; así mismo, teniendo en cuenta su lugar de origen, la intensidad luminosa pudo no ser suficiente para que el metabolismo lograra una absorción de nutrientes adecuada. Otro factor que pudo interferir en la remoción de nutrientes es el consorcio microbiano presente en la laguna, pues la relación microalga-bacterias involucra compe- tencia y depredación (Colorado \& Moreno, 2017; Ramos \& Pizarro, 2018), elementos que pueden llegar a obstaculizar la asimilación de nutrientes.

\subsection{ANOVA}

Aunque los efectos de los factores tiempo y concentración aparentan ser importantes en su representación gráfica, esto no implica que el efecto correspondiente sea estadísticamente significativo sobre las variables de respuesta. Para afirmar que tales efectos contribuyen a explicar el comportamiento de la respuesta, se debe hacer la prueba estadística del análisis de varianza (Gutiérrez \& De la Vara, 2012). Si el "Valor- $p$ " es menor que el nivel de significancia prefijado, 0.05 , se concluye que el efecto correspondiente del factor está activo o influye de manera significativa sobre la respuesta; además, mientras más pequeño sea el valor-p de un factor, este último es más importante (Gutiérrez \& De la Vara, 2012).

Los resultados del ANOBA se muestran en la tabla 6 . En el caso de la $C V$ se obtuvo un resultado inferior a 0.05 para todos los "Valor-p"; de este modo, se acepta que sí hay efecto significativo de t, C y la combinación $\mathrm{tC}$ sobre las respuestas de remoción. Las cepas $S m$ y $S p$ no registraron significancia estadística en todos los resultados, lo que se evidencia en los valores de las combinaciones $\mathrm{tC}$ de fosfatos, para $S p$, y nitratos y fosfatos para $S m$; con lo anterior se determina que dichas combinaciones no tienen un efecto significativo sobre la remoción de nutrientes, lo que permite descartar a la cepa $S p$ como biorremediadora de fosfatos. Igualmente, la Sm tampoco resulta una opción adecuada para remover nitratos y fosfatos en la laguna. 


\begin{tabular}{|c|c|c|c|c|c|c|c|c|c|}
\hline \multicolumn{10}{|c|}{ Chlorella vulgaris } \\
\hline Nutriente & \multicolumn{3}{|c|}{ Nitratos } & \multicolumn{3}{|c|}{ Nitritos } & \multicolumn{3}{|c|}{ Fosfatos } \\
\hline Fuente de variabilidad & $\mathrm{T}$ & C & $\mathrm{tC}$ & $\mathrm{t}$ & $\mathrm{C}$ & $\mathrm{tC}$ & $\mathrm{t}$ & C & $\mathrm{tC}$ \\
\hline Fo & 14.86 & 64.56 & 23.27 & 25.41 & 16.67 & 6.94 & 9.54 & 10.86 & 7.46 \\
\hline Valor-p & 0.0063 & $<0.0001$ & 0.0003 & 0.0015 & 0.0047 & 0.0337 & 0.0130 & 0.0093 & 0.0082 \\
\hline \multicolumn{10}{|l|}{ Spirulina platensis } \\
\hline Nutriente & \multicolumn{3}{|c|}{ Nitratos } & \multicolumn{3}{|c|}{ Nitritos } & \multicolumn{3}{|c|}{ Fosfatos } \\
\hline Fuente de variabilidad & $\mathrm{T}$ & $\mathrm{C}$ & $\mathrm{tC}$ & $\mathrm{t}$ & $C$ & $\mathrm{tC}$ & $\mathrm{t}$ & $\mathrm{C}$ & $\mathrm{tC}$ \\
\hline Fo & 41.91 & 35.93 & 26.49 & 238.86 & 34.75 & 70.10 & 35.98 & 29.36 & 4.70 \\
\hline Valor-p & 0.0001 & 0.0002 & $<0.0001$ & $<0.0001$ & 0.0002 & $<0.0001$ & 0.0002 & 0.0004 & 0.0584 \\
\hline \multicolumn{10}{|l|}{ Spirulina máxima } \\
\hline Nutriente & \multicolumn{3}{|c|}{ Nitratos } & \multicolumn{3}{|c|}{ Nitritos } & \multicolumn{3}{|c|}{ Fosfatos } \\
\hline Fuente de variabilidad & $\mathrm{T}$ & $\mathrm{C}$ & $\mathrm{tC}$ & $\mathrm{t}$ & $\mathrm{C}$ & $\mathrm{tC}$ & $\mathrm{t}$ & $\mathrm{C}$ & $\mathrm{tC}$ \\
\hline Fo & 6.57 & 41.18 & 2.02 & 15.95 & 17.23 & 6.00 & 14.99 & 10.30 & 3.68 \\
\hline Valor-p & 0.0306 & 0.0001 & 0.1892 & 0.0031 & 0.0025 & 0.0368 & 0.0038 & 0.0107 & 0.0872 \\
\hline
\end{tabular}

Tabla 6. Resultados del ANOVA.

En las tres cepas, los "Valor- $p$ " de magnitud tan pequeña muestran que la significancia es contundente y que prácticamente no se corre ningún riesgo en aceptarla. La cepa $C v$ fue la que demostró la mejor significancia estadística sobre la remoción de nutrientes.

Al igual que el "Valor-p", el "Valor Fo" permite determinar la importancia de un determinado factor sobre las respuestas del diseño, entre más alto sea el valor, el efecto e importancia del factor será mayor (Gutiérrez \& De la Vara, 2012). Para las cepas $C v$ y $S m$ el factor más influyente fue la concentración; por el contrario, para la $S p$ el tiempo de retención resultó más importante; lo anterior se tuvo en cuenta para la optimización numérica de cada cepa.

\subsection{Optimización numérica}

La tabla 7 muestra la optimización numérica producto del diseño experimental en Design Expert; en ella se evidencian las alternativas con las cuales es posible obtener resultados de remoción significativos para todos los nutrientes. En la obtención de dichas alternativas no solo se consideró la eficacia, sino también la eficiencia en términos de tiempo retención y concentración, con el fin de optimizar costos de implementación. 


\begin{tabular}{llll}
\hline Variables & \multicolumn{3}{l}{ Alternativas } \\
\hline & $\mathrm{Cv}$ & $\mathrm{Sp}$ & $\mathrm{Sm}$ \\
$\mathrm{T}$ & 3.13 & 4.13 & 3.84 \\
$\mathrm{C}$ & 12.89 & 15.05 & 16.24 \\
\% Remoción NO3- & 87.00 & 54.00 & 51.00 \\
\% Remoción NO2- & 89.00 & 57.88 & 70.00 \\
\% Remoción PO43- & 69.00 & 68.85 & 88.00 \\
Deseabilidad & 0.766 & 0.568 & 0.565 \\
\hline
\end{tabular}

Tabla 7. Optimización numérica.

\section{Conclusiones}

La biorremediación con microalgas es una alternativa efectiva para remover los nutrientes, $\mathrm{NO} 3$ -, NO2 - y PO4 -3 de la laguna de Ubaque; la cepa más apta para la aplicación del proyecto es la Chlorella vulgaris, la cual demostró una mayor capacidad de adaptación a las condiciones de la laguna y presentó una remoción superior de los nutrientes. Se recomienda que en el proceso de biorremediación, la cepa $C V$ sea cultivada a una concentración V/V de $12.89 \%$ en un tiempo de retención de 3.13 días, con el fin de obtener resultados de remoción eficientes y eficaces.

Como consecuencia de la presente investigación, se sugiere la realización de posteriores estudios enfocados a llevar éste experimento conceptual a lo aplicado. En este sentido, se recomienda la construcción de un sistema de cultivo abierto en el cual la microalga pueda estar expuesta a las condiciones de la laguna, para lograr su adaptación antes de ser utilizada en la remoción de nutrientes.

La interacción microalga/bacteria en la laguna de Ubaque no fue evaluada en el presente estudio, pero teniendo en cuenta que pudo ser un factor determinante en la asimilación de nutrientes, se sugiere su estudio en investigaciones futuras.

\section{Referencias}

Álvarez, P. (2014). Fotobiodepuración de aguas residuales optimización de la producción de microalgas con elevado contenido lipídico. (Tesis de pregrado). Universidad de Cádiz. Cádiz, España.

Ávila, H. (2006). Introducción a la metodología de la investigación. Chihuahua, México: Editorial Eumed.

Bermeo, L. (2011). Estudio del cosechado de cultivos de microalgas en agua residual mediante técnicas de centrifugado. (Tesis de pregrado). Universidad de Cádiz. Cádiz, España.

Blanch, D., Cárdenas, M., Durnin, S., Herrera, X., \& Ortiz, A. (2017). Biorremediación a través de dos diferentes especies de microalgas, a partir de la presencia de ciertos metales pesados. RPubs, 1, 1-8. Recuperado de: https://rpubs.com/MeliCF/280674

Bolaños-Ortiz, S. S., \& Martínez-Caranguay, G. N. (2016). Producción de lípidos a partir de la microalga Chlorella vulgaris. UNIMAR, 1, 345-354. Recuperado de: http://www.umariana.edu.co/ojs-editorial/index.php/libroseditorialunimar/article/ view/976

Carbiener, R., Trémolieres, M., Mercier, J.L., \& Ortscheit, A. (1990). Las comunidades de macrófitos acuáticos como bioindicadores de la eutrofización 
en las aguas de la corriente de oligosaprobe calcárea (Alto Rin, Alsacia). Vegetatio, 86, 71-88.

Carmona, A., \& Cruz, A. (2014). Determinación del costo - beneficio del proceso de descontaminación de la laguna de Ubaque (Cundinamarca) a partir de la aplicación del método de la valoración contingente. (Tesis de pregrado). Universidad Libre, Bogotá, Colombia.

Cartagena, J. C., \& Malo, B. O. (2017). Evaluación del uso de la microalga Chlorella vulgaris en la remoción de Materia Orgánica de las Aguas Residuales de la PTAR EI Salitre a nivel laboratorio. (Tesis de pregrado). Universidad de América, Bogotá, Colombia.

Castellanos, N., \& Charry, M. (2016). Evaluación de la Calidad Físico-Química y Biológica de la Laguna de Ubaque para el Diseño y Actualización de las Medidas de Manejo Ambiental. (Tesis de pregrado). Universidad Libre, Bogotá, Colombia.

Colorado-Gómez, M. A., \& Moreno-Tirado, D. A. (2017). Economía de Recursos Naturales a partir de la Producción de Spirulina (Arthrospira maxima) en Fotobiorreactores, La Guajira, Colombia. RETO, 5, 50-59.

Desing Expert. (2018). Diseños de Superficie de Respuesta. Recuperado de: https://www.statease. com/docs/v11/designs/rsm.html\#rsm.

Dzionek, A., Wojcieszynkska, D., \& Guzik, U. (2016). Portadores naturales en biorremediación: una revisión. Electronic Journal of Biotechnology, 23, 2836. doi: 10.1016/j.ejbt.2016.07.003

Forero-Cujiño, M., Montenegro-Ruíz, L., PiniIla-Agudelo, G., \& Melgarejo-Muñoz, L. (2015). Inmovilización de las microalgas Scenedesmus Ovalternus (Scenedesmaceae) Y Chlorella Vulgaris (Chlorellaceae) en esferas de Alginato de Calcio. Acta Biológica Colombiana, 21, 437-442. doi: https://doi.org/10.15446/abc.v21n2.51253
Gutiérrez, H. \& De la Vara, R. (2012). Análisis y Diseño de Experimentos. México: Editorial Mc Graw Hill.

Ilzarbe-Izquierdo, L., Tanco, M., Viles, E. \& Álvarez, M. J. (2007). El diseño de experimentos como herramienta para la mejora de los procesos. Aplicación de la metodología al caso de una catapulta. Tecnura, 10, 127-138. doi: https://doi. org/10.14483/22487638.6254

Instituto de Hidrología, Meteorología y Estudios Ambientales. (IDEAM, 2017). Guía para el monitoreo de vertimientos, aguas superficiales y subterráneas. Recuperado de: http://corponor.gov.co/ corponor/sigescor2010/TRAMITESYSERVICIOS/ Guia_monitoreo_IDEAM.pdf

Instituto de Hidrología, Meteorología y Estudios Ambientales. (IDEAM, 2018). Metodologías de Análisis. Recuperado de: http://www.ideam.gov.co/ web/agua/metodos-analiticos

Malgas. (2013). Aplicaciones de las microalgas: estado de la técnica. Recuperado de: http://proyectomalgas.com/wp-content/uploads/2014/04/guiamalgas.pdf.

Medina, P., \& López, A. (2011). Análisis crítico del diseño factorial $2 \mathrm{k}$ sobre casos aplicados. Scientia Et Technica. 47, 101-106. Recuperado de: http:// www.redalyc.org/articulo.oa?id=84921327018

Mehrabadi, A., Craggs, R., \& Farid, M. (2015). Tratamiento de aguas residuales de alta tasa de estanques de algas (WWT HRAP) para la producción de biocombustibles de bajo costo. Biosurce Technology, 184, 202-214. doi: 10.1016/j.biortech.2014.11.004

Ministerio de Ambiente y Desarrollo Sostenible. (2016). Política Nacional para la Gestión Integral de la Biodiversidad y sus Servicios Ecosistémicos. Recuperado de: http://www.humboldt.org.co/images/ pdf/PNGIBSE_espa\%C3\%B1ol_web.pdf 
Moreta, J. (2008). La Eutrofización de los Lagos y sus Consecuencias. (Tesis de pregrado). Universidad Técnica del Norte, Ibarra, Ecuador.

Mosa, K., Saadoun, I., Kumar, K., Helmy, M., \& Dhankher, O. P. (2016). Posibles estrategias biotecnológicas para la limpieza de metales pesados y metaloides. Frontiers in Plant Science, 7, 303. doi: 10.3389/fpls.2016.00303

Pozo, J. (2011). Puesta en marcha de un reactor aerobio de lecho fluidizado para la eliminación de nitrógeno amoniacal. Bogotá, Colombia: Editorial Lulu.

Ramos, R., \& Pizarro, R. (2018). Crecimiento y capacidad de biorremediación de Chlorella vulgaris (Trebouxiophycea, Chlorophyta) cultivada en aguas residuales generadas en el cultivo del pez dorado Seriola lalandi (Perciformes: Carangidae). Revista de Biología Marina y Oceanografía, 53, 7586. Recuperado de: http://revbiolmar.cl/resumenes/v531/v531a/531-75.pdf

Rawat, R., Ranjith, T., Mutanda, F., \& Bux F. (2011). Doble función de las microalgas Fitorremediación de las aguas residuales domésticas y la producción de biomasa para la producción sostenible de biocombustibles. Applied Energy, 88, 3411-3413.
Rodríguez, R., Espada, J., Moreno, J., Vicente, G., Bautista, L. F., Morales, V., Sánchez-Bayo, A., \& Dufour, J. (2017). Análisis ambiental del cultivo de Spirulina y la producción de biogás utilizando un enfoque experimental y de simulación. Renewable Energy, en prensa, prueba corregida. doi: 10.1016/j. renene.2017.05.076

Rodríguez-Miranda, J. P., Serna-Mosquera, J. A., \& Sánchez-Céspedes, J. M. (2016). Índices de calidad en cuerpos de agua superficiales en la planificación de los recursos hídricos. Revista Logos Ciencia \& Tecnología, 8, 159-167. Recuperado de: http:// revistalogos.policia.edu.co/index.php/rlct/article/ view/306

Universidad Tecnológica de Panamá. (2006). Procedimiento para la Prueba de Nitrito. Recuperado de: http://www.utp.ac.pa/documentos/2011/pdf/ PCUTP-CIHH-LSA-208-2006.pdf.

Weizhi, Z., Yating L., Yizhan, G. \& Haixia, Z. (2017). Eliminación de nutrientes y recuperación de aguas residuales salinas por Spirulina platensis. Bioresource Technology, 245, 10-17.

Zehnsdorf, A., Hussner A., Eismann, F., Ronicke H., \& Melzer, A. (2015). Opciones de manejo invasivo de Elodea nuttallii y Elodea canadensis. Limnologica, 51, 110-117. 